\title{
Chapter 3 \\ The Path to Scale: Navigating Design, Policy, and Infrastructure
}

\author{
Jay Evans, Shreya Bhatt and Ranju Sharma
}

\begin{abstract}
Health offers a unique opportunity to improve access, quality, and adherence of care in last mile and low-resource settings around the world. However, the path to scale for mHealth interventions can be complex and challenging due to the barriers presented by fragmented infrastructure, policy gaps, and more. This chapter proposes a framework of nine key components that are essential for the successful scale-up of mHealth including mature infrastructure, a conducive policy environment, strong institutional partnerships, well-designed and contextappropriate technology, a skilled health workforce, financial sustainability, interoperability, and an evidence-based approach to mHealth. While not exhaustive, this framework offers implementers and policymakers a potential path to scale up mHealth interventions in order to strengthen health systems and improve health outcomes - particularly in remote communities around the world.
\end{abstract}

Keywords Scale • Policy • Infrastructure $\cdot$ Human-centered design

Cost-effectiveness $\cdot$ Data security $\cdot$ Integration $\cdot$ Interoperability

\subsection{Introduction}

mHealth has unearthed a unique opportunity to improve the delivery of healthcare in the most disconnected and remote regions of the world. Nowhere is this more pronounced than in low- and middle-income countries (LMICs) where health systems often face many challenges and require innovative solutions to strengthen these systems and improve health outcomes (Mills, 2014). Leveraging mobile

J. Evans $(\bowtie) \cdot$ R. Sharma

Medic Mobile, Kathmandu, Nepal

e-mail: jay@medicmobile.org

J. Evans

Global Health Academy, University of Edinburgh, Edinburgh, UK

S. Bhatt

Medic Mobile, Mumbai, India

(C) Asian Development Bank 2018

E. Baulch et al. (eds.), mHealth Innovation in Asia, Mobile Communication in Asia:

Local Insights, Global Implications, https://doi.org/10.1007/978-94-024-1251-2_3 
technology to address these burgeoning health systems challenges, the field of mHealth has experienced a gold rush in recent years with more than 500 distinct pilots implemented globally, many of which are in developing countries (Bjornland, Goh, Haanæs, Kainu, \& Kennedy, 2012).

Despite the enthusiasm around mHealth, pathways to scale remain fraught with challenges. After the completion of hundreds of pilots, not enough is known about the uptake, efficacy, and effectiveness of mHealth interventions (Tomlinson, Rotheram-Borus, Swartz, \& Tsai, 2013). Evidence shows that few mHealth pilots successfully upscale and that most often expire once the initial project funding is exhausted, with project smartphones and tablets relegated to shelves and warehouses to gather dust (Lemaire, 2011). In Uganda, for example, roughly 64\% of mHealth interventions piloted in 2008 and 2009 failed to move beyond pilot stage (ibid.). There are lessons to be learned from those mHealth initiatives that have scaled sustainably which could be applied to the early stages of planning and design for scale (Hall, Fottrell, Wilkinson, \& Byass, 2014). As mHealth pilots continue to grow and change the way healthcare is delivered in specific contexts, there is a pressing need for a deeper and more nuanced understanding of pathways to scale, particularly the challenges that hinder growth. For example, the Digital Development Principles Working Group provides a set of guidelines for technology-enabled programs proposed by a consortium of global organizations including United Nations agencies that can help to shape future mHealth programs (Waugaman, 2016).

In this chapter, we draw upon our own experience at Medic Mobile of deploying mHealth projects in over 23 countries in Asia and Africa, as well as available literature that supports the use of best practices for mHealth scale. Medic Mobile is a nonprofit technology company with offices in San Francisco, Nairobi, and Kathmandu that builds mobile and web tools for health workers, helping them provide better care that reaches everyone. Medic Mobile develops open-source tools that can be adapted for specific uses, backed by evidence. Medic Mobile works with implementing partners such as ministries of health and international and local nonprofit organizations, to deploy projects that leverage mHealth to improve health in last-mile settings around the world.

mHealth interventions are usually complex and their success depends upon the confluence of several factors and functions, in the absence of which scale becomes seemingly unattainable. These factors can be thought of as prerequisites and can be distilled into nine key components: Infrastructure, Policy, Institutional Partnerships, Technology, Interoperability, User-centered Design, Financial Sustainability, Human Resources, and Impact, that collectively make a "framework" for scale (see Fig. 3.1). While this is not an exhaustive list, it provides a framework to understand the challenges faced by mHealth designers and implementers in scaling mHealth interventions and hidden opportunities that may be leveraged to overcome these hurdles in an effective manner. 


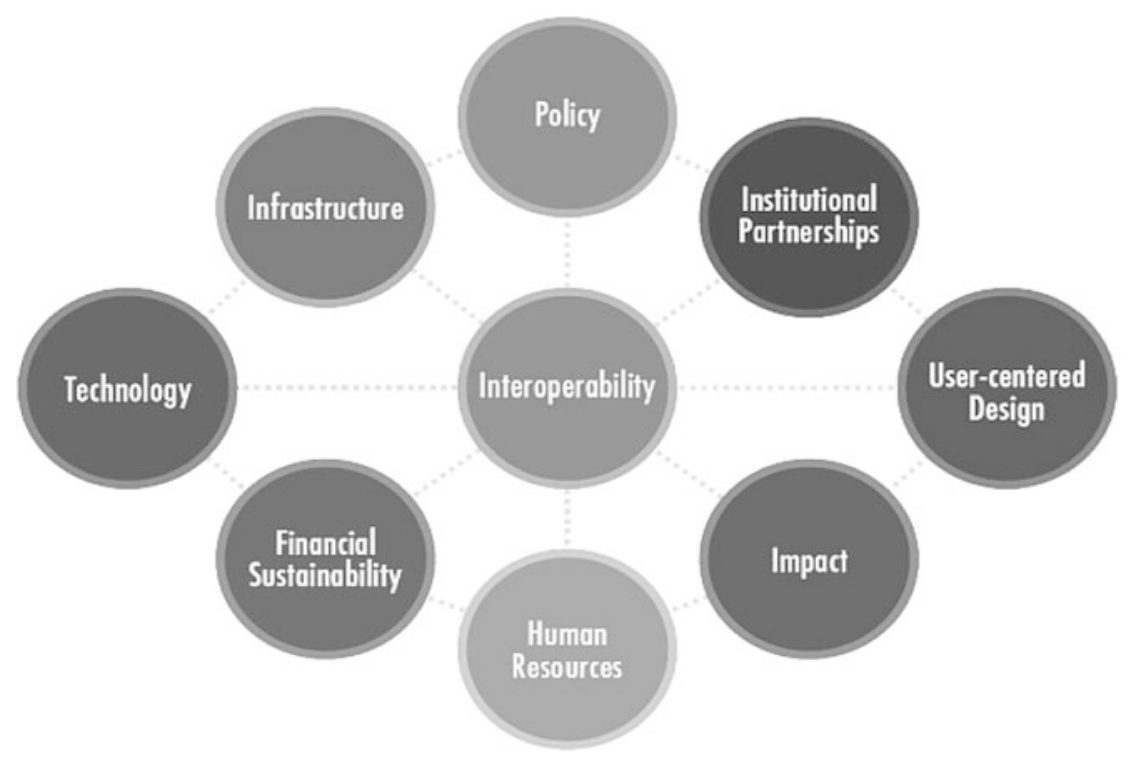

Fig. 3.1 Framework for mHealth scale

\subsection{Infrastructure: Creating a Balance Between Feasibility and Sustainability}

Infrastructure is one of the principal challenges to scale and sustainability of mHealth initiatives around the world, particularly in low-resource settings (Aranda-Jan, Mohutsiwa-Dibe, \& Loukanova, 2014; Umali, McCool, \& Whittaker, 2016). mHealth interventions are highly dependent on the available infrastructure, especially with regards to network coverage, supply of electricity, and internet access. Even though mobile network coverage is reported to cover close to $97 \%$ of the global population (International Telecommunications Union, 2015) internet access remains limited in many regions. Only $69 \%$ of the global population enjoys $3 \mathrm{G}$ mobile-broadband coverage and nearly $66 \%$ of the population in developing countries and $90 \%$ in least-developed countries continue to remain offline. Given the limited access in low-resource settings, mHealth interventions that do require internet connectivity for smartphones and tablets are more prone to challenges in implementation as well as limitations in their scalability (Ngabo et al., 2012).

In some countries, more people have access to a cell phone signal than to electricity (Kay, Santos, \& Takane, 2011). For example, the cell network in Nepal covers roughly $97 \%$ of the population (World Bank, 2016a) but only $76 \%$ of the population has access to electricity (2016b). The population in Nepal that does have access to electricity during the dry winter season enjoys as little as 8 hours of power per day due to rationing and those hours may be distributed during nonoptimal 
times such as working hours or in the middle of the night (World Bank, 2011). The limited availability of electricity and its "usability" - e.g., the hours when a user can actually charge their phone - is often cited as a challenge faced by mHealth users in low-resource settings and should be considered carefully when selecting hardware during the initial design of mHealth initiatives (Chang et al., 2011). Furthermore, users may have only one or two available power outlets in their homes for all household electrical devices and as a result, they have to compete for access to these outlets with other devices and family members. In the event of limited access to electricity, mHealth users have sought other means to charge their devices such as traveling to local shops or small business, which incurs costs of travel and charging fees (Thondoo et al., 2015). In response to these challenges of access and affordability of power, users often voice a preference for devices that maximize battery life even at the cost of foregoing more advanced and multifunctional devices in favor of a simple phone (ibid.). Devices that can be easily charged within a few hours and retain that charge for longer periods will most likely have greater uptake and prove more successful in the long-term. Cheaper alternatives to charging devices such as using car batteries or solar chargers have been shown to be effective in various contexts and should be explored as potential solutions to the challenges of limited electricity in low-resource settings (Chang et al., 2011; Thondoo et al., 2015).

Our experience has shown the importance of identifying infrastructure-related challenges and referring to them during the designing and implementation of mHealth interventions. In the aftermath of the 7.8 Richter scale earthquake in Nepal in April 2015, Medic Mobile in collaboration with the Ministry of Health in Nepal deployed an mHealth intervention for daily reporting and suspected outbreak reporting of epidemic-prone diseases. The intervention was deployed in hospitals in both the urban Kathmandu valley and the heavily hit remote district of Dhading (Nesbit, 2015). The infrastructure available in these sites are very different and therefore, designing a "one-size-fits-all" mHealth tool would simply not work. While Kathmandu is a major urban center with reliable mobile phone coverage, adequate electricity for charging smartphones and easily accessible facilities for maintenance and repair of smartphones, Dhading is fairly remote with limited infrastructure. Moreover, the earthquake destroyed or severely damaged most shelters - including health facilities - in Dhading district, where $85 \%$ of the facilities were rendered non-functional according to Nepal's Child Health Division (Khanal Khanal \& Lee, 2015). Road access to northern parts of the district was also cut off (Adhikari, 2015). In light of these challenges, the intervention had to be redesigned before implementation in Dhading, including the mHealth tool itself. While the community health volunteers in Kathmandu valley were able to use an Android application with internet connectivity, those in Dhading used basic phones and structured SMS, which were all that could be supported by the low-infrastructure settings of the district weakened even further by the earthquake. Health facilities in the northern areas which did not have GSM or CDMA connectivity following the earthquake submitted SMS-based reports using CDMA landline phones. Moreover, the structure of the forms on the mobile devices was 
also designed in such a way that it required minimal training, as health workers were trained remotely via phone calls due to the urgency of the situation and the inaccessibility of many areas. Therefore access to reliable, stable electricity and internet remain major obstacles to scale for mHealth tools that rely solely on higher end smartphone and apps requiring frequent connectivity and greater infrastructural support. Resolving these challenges requires careful design and selection of both the hardware and software to be deployed.

\subsection{Technology: The Right Tools for the Right Context}

The selection of hardware also shapes the future scale of mHealth interventions and can pose a challenge for the long-term sustainability of projects if not planned and executed well. Many times when launching an mHealth initiative, technological wizardry or the "bells and whistles" of new and rapidly evolving technology shape the deployment and implementation pathway rather than user needs and health priorities (Shaw, 2012). Hardware selection can also become unduly influenced by procurement and contracting guidelines created by staff unfamiliar with the limitations of settings in LMICs (Bernhardsen, 1999). Not only are newer technologies often unsupported by reliable access to network, internet, and electricity that evolve at a slower pace, but oftentimes they are not context-appropriate. Hardware used by health workers particularly in rural areas of the developing world is prone to experience excessive wear and tear over time (Iluyemi \& Briggs, 2008). Ensuring the growth and long-term sustainability of mHealth initiatives then necessitates that the chosen devices can be easily replaceable or repairable in the areas where they are deployed. Corner cell phone shops or local kiosks that are ubiquitous in most small villages and towns around the world typically serve as the first point of contact for access to basic mobile hardware and repair services (Chang et al., 2011). When considering hardware for deployment in an mHealth intervention, these shops - and the devices they sell—-should serve as a yardstick for the appropriate selection of project hardware. When mHealth interventions employ devices that are foreign or not easily available in local markets, they are by default harder to repair or replace in the event of damage or loss and may cause a reduction in user engagement. It is, therefore, crucial to use context-appropriate hardware that can be easily procured and repaired close to where users are located to ensure the future scale and sustainability of the intervention.

Hardware selection not only involves making decisions about the right tools for the context, but also about whether to use devices already owned by the users or provide new devices for the duration of the mHealth intervention (Ben-Zeev et. al., 2015). Benefits of using existing phones owned by users include greater user familiarity with the device and a higher likelihood that they will intuitively understand how to use the tool. However, relying on existing devices also poses several challenges. Phones owned by users may differ in their features, functionalities, carriers, and data plans. They may also be ill-suited to the specific needs of 
the intervention. While providing new devices to users can help to standardize the intervention across all users, it may also decrease the frequency with which the new devices are used, cause negative user experiences from having to use multiple devices for different purposes and require more user trainings, not to mention the financial implications of purchasing devices at scale for hundreds or thousands of users. New devices can also act as an incentive for users, particularly if they are allowed to keep the device after completion of the intervention, however, this may not always be feasible (ibid.).

Another factor that can influence this decision is the variance in mobile phone ownership amongst users. Not all users such as care providers and patients own a personal mobile device and often share mobile phones with family members or access the phones available to them in their community (Chang et al., 2011; Haberer, Kiwanuka, Nansera, Wilson \& Bangsberg, 2010). Moreover, phone ownership rates vary significantly based on gender particularly in the developing world. There are 200 million fewer female mobile phone subscribers than male subscribers in LMICs and many women in these settings only have partial access to a mobile phone owned by male members of their family during nonbusiness hours such as evenings (GSMA, 2015). These early decisions about hardware selection based on phone ownership and suitability for context shape the mHealth intervention and can have long-lasting implications on the uptake, success, and scale of mHealth interventions.

\subsection{Institutional Partnerships}

Literature has extensively highlighted the importance of strong cooperation between various actors such as government, funders, and private enterprise for mHealth scale (Tomlinson et al., 2013; Qiang, Yamamichi, Hausman \& Altman, 2011). These partnerships serve to incentivize and reinforce positive behaviors and are vital to create a conducive ecosystem in which mHealth can flourish. The role of mobile network operators (MNOs) in scaling mHealth interventions is particularly important (Qiang et al., 2011; Sanner, Roland \& Braa, 2012). MNOs provide the architecture for implementing mHealth interventions, and as such their cooperation is crucial to the success of an mHealth deployment. However, when creating alliances with an MNO, their market share and acceptability within user populations in rural and urban areas should be taken into consideration - particularly in countries where the telecommunications industry is highly diversified (Qiang et al., 2011). The MNO landscape in India, for example, is highly competitive with more than ten MNOs offering network coverage in most parts of the country and no single MNO dominating the market. Given the competition in such settings, users frequently switch between operators in favor of those that offer the most minutes or SMS at the lowest price (Airtel, 2016). Therefore, choosing a single MNO partner for mHealth across the country in such settings becomes difficult. 
A potential alternative to MNO partnerships may be to lobby for in-kind services from MNOs by leveraging government or physician membership organization relationships. An example of this is Switchboard, a nonprofit organization that has successfully created a free calling network for healthcare providers in Ghana and Liberia (Switchboard, 2017). The network not only benefits health systems in these countries by facilitating free communication and coordination between care providers in rural and urban health centers, but also serves to build MNO brand loyalty among users and increases the participating MNOs' consumer base as healthcare providers increasingly use the same operators for their personal use. Incentivizing MNOs to dedicate free or reduced cost SMS, data, and call facilities may be a more effective long-term strategy for mHealth scale and sustainability rather than relying on partnership agreements with a single operator. Innovative partnerships that create incentives and positively change stakeholder behaviors, therefore, are crucial for the success and scale of mHealth interventions.

\subsection{Human Resources}

The crucial components of infrastructure for mHealth scale and sustainability are not just limited to the availability of context-appropriate hardware, a cell phone tower, and an active power source but also include human resource infrastructure in the form of a skilled health workforce. In many LMICs, the cadre of community health workers (CHWs) may have achieved only basic literacy levels via formal schooling and typically have no tertiary education (Lehmann \& Sanders, 2007). Health professionals need to be educated on the potential role of technology in healthcare delivery in order to achieve and sustain mass adoption of mHealth (Mechael et al., 2010). While CHWs in LMICs receive standard training on topics such as general health and basic record-keeping, such training should not be assumed to include indoctrination into mHealth (Lehmann \& Sanders, 2007). CHWs and other healthcare personnel may already own and be familiar with using cell phones but they may not be well-versed with specific features and are often not fully prepared or equipped to use those same devices for an mHealth project (Ben-Zeev et al., 2015). In order for CHWs to start thinking of and using their mobiles as communication and coordination tools for health activities that form part of a ministry of health information system, they must have adequate and context-relevant training in technology (Mechael et al., 2010). Given the variance in skill sets and levels of literacy among CHWs, there is a need for continuous support and training to maintain their effective contribution not only in their healthcare knowledge but also in technology and its use (Lehmann \& Sanders, 2007). Refresher mHealth training for CHWs have been effective in addressing observed technology usability gaps and improving impact during an intervention and can also contribute significantly to long-term project sustainability and scale (Modi et al., 2015; Haberer et al., 2010). mHealth training is not only essential for users such as healthcare providers but also for other key actors in an mHealth ecosystem such as 
project managers, application developers, and information technology specialists who require training in the development and maintenance of platforms including software and hardware to support mHealth implementations locally (Chetley, Davies, Trude, McConnell \& Ramirez, 2006; Aranda-Jan et al., 2014). Therefore, a health workforce skilled in the use and maintenance of technology is a prerequisite for the success and scale of technology interventions.

A related barrier to the scale of mHealth interventions is continued engagement and motivation of health workers over the duration of the project and in the longer run. While users may be motivated at the start of a project, experience suggests that this motivation may often wane over longer periods of time (Haberer et al., 2010). Although research on CHW motivation as a result of mHealth is limited, there is some evidence to suggest that being more efficient and effective in their work tasks by virtue of mHealth is motivating for CHWs (Strachan et al., 2012). To the extent that mHealth interventions can continue to enhance health worker efficiency, CHWs will remain motivated to engage with the technology platforms for longer periods of time. Beyond efficiency, CHWs also respond to a sense of being valued by the communities they serve and the health systems they work within. A motivational technique that the mHealth system in Nepal uses, for instance, is a simple "thank you" text message delivered to CHWs after they perform certain activities such as registering a new pregnancy on their mobile device; this has been found to improve and sustain CHW motivation levels over time (Sharma et al., 2015).

Experience also shows that mHealth training for CHWs and the resulting improvement in their skill set leads to greater health worker confidence, motivation, and enthusiasm to participate in mHealth interventions (Haberer et al., 2010). Continuous training during mHealth deployments, therefore, is important to not only update knowledge and skills but also to maintain health worker enthusiasm levels throughout the duration of the project and beyond. Finally, several mHealth initiatives have also found that providing minor financial incentives such as airtime credit motivates CHWs and ensures their continued participation and uptake of technology (ibid.; Lester et al., 2010). When planning and designing for scale, mHealth interventions that can budget for such incentives for health workers over the life of the deployment stand a greater chance of long-term CHW engagement and therefore success.

\subsection{Policy}

The national policy and regulatory landscape - particularly an LMIC — is essential for mHealth scale and sustainability. The lack of guiding policies from the government is frequently cited as a major reason for the failure of mHealth programs (Leon, Schneider \& Daviaud, 2012; Aranda-Jan et al., 2014). When an LMIC government defines an mHealth and wider eHealth strategy-including clearly established standards for data security and interoperability-and is willing to facilitate the integration of mHealth initiatives into the existing healthcare system, 
the mHealth systems in that country are more likely to be sustained (Aranda-Jan et al., 2014; Lemaire, 2011). While a significant proportion of countries have recognized this and more than half of WHO member states have already adopted a broader eHealth strategy within which national mHealth programs can be ensconced (World Health Organization, 2016) much more remains to be done. In a recent WHO survey, the lack of legal regulation was cited as one of the top two barriers to mHealth (ibid.). Much of the need for legal regulation around mHealth in LMICs stems from data privacy and confidentiality concerns.

Traditional paper-based systems of healthcare inherently pose a data privacy risk which can be mitigated by electronic health records and care coordination systems such as mHealth. As a result, mHealth and the broader eHealth ecosystem are often framed as "safe" mechanisms to facilitate and provide health service delivery. However, governments must define and impose comprehensive legal provisions to ensure that the storage and exchange of information over electronic methods are truly safe, particularly, in LMICs where mHealth platforms are already beginning to flourish. Legislation alone, however, is not the solution. Experience has shown that even when mHealth legislation exists, public misinformation may, in fact, derail an mHealth project (Eysenbach, 2009). Therefore, governments not only need to create and implement appropriate legislation, but also put in place a regulatory authority or body to monitor mHealth initiatives within a country. In the absence of both clearly established legislation and a regulatory authority, the integrity and credibility of any new mHealth tool may be jeopardized.

While government institutions have programmatic authority to evaluate mHealth initiatives, the lack of legislative authority with the knowledge to execute fair judgment may render their mHealth evaluations baseless and ineffective, hindering the scale of such programs. Moreover, the interpretation of the newly passed regulations may face challenges in courts where judges presiding over such cases may not have sufficient experience adjudicating cases related to technology (Timm, 2014). In such cases, a regulatory authority overseeing mHealth programs may be able to lend their expertise and knowledge to bring such cases to a just culmination. It is equally important to note that while some states have passed complete sets of regulations that are intended to govern $\mathrm{mHealth}$ within their borders, there are many times poor coordination among competing government ministries and agencies in charge of the oversight and management of the mHealth space (Lemaire, 2011). The same can be true among different departments managing various verticals within a ministry of health. Given such challenges, the mHealth authority in the country must also take on the coordination around policy and legislation among relevant ministries and sectors (ibid.).

The mHealth landscape in a country is not only affected by its own policy and regulatory environment, but also by the policy and regulatory settings of its neighbors the surrounding region. Policies and legislation on mHealth in LMICs, as well as developed countries, tend to vary a great deal. For example, regulations on spam advertising to mobile phones and privacy policies for smartphone apps-as well as what these policies are allowed to include - often vary significantly from country to country even within the same region such as South Asia or East or West 
Africa. Significant variations exist in policies and regulations around mHealth even in countries belonging to the same geographical region, which may negatively affect mHealth scale across borders (World Health Organization, 2016). Moreover, few standards exist for data confidentiality and sharing among countries within geographical regions, which further hinders mHealth scale across borders (ibid.).

In an ideal scenario, all relevant mHealth-related policies are well-established and institutionalized by the time an mHealth initiative is ready to scale. However, the current reality is that many LMICs are still in the process of developing or refining such policies, and the speed of maturity of mHealth initiatives and supporting public policies do not match. Even if the framework legislation has been established, the regulatory environment that must accompany laws on mHealth is simply not present in many LMICs. Given this, mHealth systems must also, for the time being, address such gaps. Aligning an mHealth tool to fit within the confines of a nascent regulatory structure means that the tool must also be flexible enough to change and adapt to new regulations as they emerge. At times this may mean establishing the capacity for ongoing design evaluation during or after a successful small-scale pilot of the tool. Deploying within a country where the regulatory framework around mHealth may not be fully developed will also demand more time dedicated to building and maintaining relationships with the Ministry of Health and other ministries involved in the governance of digital health. Failure to actively engage government ministries may result in projects being delayed, abandoned, or outright banned (Eckman, Gorski \& Mehta, 2016).

mHealth scale cannot be achieved in the absence of an effective policy and regulatory environment and there are some immediate steps that can be taken to achieve this. LMIC governments must adopt appropriate legislation and establish a regulatory authority to create a framework within which mHealth initiatives can flourish. Simultaneously - at the global community level—international efforts must focus on identifying best policy practices that enable and promote mHealth adoption and innovation particularly in low-resource settings (World Health Organization, 2016).

\subsection{Financial Sustainability}

\subsubsection{Direct Government Financing}

Lack of funding is one of the top reasons for the premature discontinuation of potentially valuable mHealth initiatives (World Health Organization, 2016). Moreover, empirical evidence also suggests that health technology projects often cost more than initially planned, imposing additional financial pressures during the life of a project (Leon et al., 2012). In most countries, government funding commitment to an mHealth initiative is critical to ensuring its continuity as an integrated 
component within an existing health system. As discussed above, well-established mHealth governance mechanisms, comprehensive evidence to supporting the mHealth system and ground level consensus on the value and credibility of the system are all critical in guaranteeing government funding. Furthermore, the timelines for national planning and budgeting need to be considered and planned for accordingly. The government needs to be prepared to propose new inclusions into national budgets well before the new fiscal year is set to begin or any periodic plans are being formulated. Even though a national health budget might be governed by a health ministry, the budget requires approval from a finance ministry. Hence communication between the health and finance ministries is critical to the budget approval process and this requires the health ministry to speak the same language as their colleagues in finance; just being enthusiastic about a new mHealth initiative will not be sufficient.

\subsubsection{Alternative Ways of Sustainable Financing}

Financing does not only imply assigning budgets for required line items. There are various ways that a government might finance any mHealth initiative - getting subsidies for SMS, data, and voice calls is one of the most relevant ways. Government is usually the most appropriate agency to request MNOs in a given country to provide subsidies for mHealth initiatives; such a request is easiest for MNOs to process when it involves specific professional groups of health workers. An example of this is the partnership between the Rwandan Ministry of Health and MNOs that resulted in a ten-fold reduction in the cost per SMS for a mHealth pilot to improve maternal and child health in the country, which was crucial in planning for the project's expansion and ensuring its long-term sustainability (Ngabo et al., 2012).

Building components of mHealth foundational and continuing training into an existing national curriculum for health workers can be an efficient way to finance a major component of most mHealth initiatives. Operational costs such as personnel salaries and initiatives, hardware and software maintenance or update can also be absorbed into regular program budgets. In addition, buy-in and ownership of the community that is most intimately impacted by the mHealth initiative may attract some portion of direct funding as well as provide evidence of system uptake.

\subsubsection{Cost-Effectiveness}

A major barrier to scale for mHealth interventions is their perceived cost-effectiveness versus paper-based systems that they are intended to replace. Insufficient evidence exists about the cost-effectiveness of mHealth interventions as many evaluations 
focus on feasibility and user acceptance rather than cost. Where information on costs is available, it can be limited and difficult to interpret due to subsidies in technology (Zurovac et al., 2011; Leon et al., 2012). Designing for scale and sustainability requires an understanding of the various elements that make up the total cost of an mHealth intervention including developing and maintaining platforms, training and retraining users, procuring and replacing hardware as well as ongoing data and SMS costs. Opportunities exist in each of these elements to improve the overall cost-effectiveness of an mHealth intervention, particularly at scale.

When it comes to developing systems, leveraging open-source platforms that are freely available and reusable rather than proprietary systems help to lower costs significantly, especially for future redesign, implementation, and scale (Rajput et al., 2012). Hardware costs can be a particularly daunting challenge. While the costs of smartphones in both developed and LMIC markets are rapidly declining making advanced mobile devices more widely available to greater proportion of the population (The Economist, 2014), procuring handsets at scale can pose a significant initial investment and requires financial support and subsidies to enhance cost-effectiveness of the intervention (Qiang et al., 2011). Moreover, mobile devices are often prone to theft, loss or damage in LMIC settings and costs to repair or replace devices at scale can be prohibitive (Chang et al., 2011). Strategies to curb costs of purchasing new devices may include using locally available entry-level phones (Leon et al., 2012), designing flexible, device-agnostic systems that can work on a range of mobile devices and facilitate the selection of least expensive hardware for future implementation (Rajput et al., 2012) or leveraging personal phones of users, keeping in mind the potential shortcomings of such a choice. The ongoing costs of mHealth intervention include data, voice, and SMS charges and while these rates can be quite low in many LMICs, significant budgeting and funding is required to meet these ongoing cost components at project scale where thousands of users are required to send text messages or make calls on a daily basis. Hence the importance of strategies which lower these ongoing costs such as private-public partnerships between government and private MNOs as mentioned earlier.

A frequent question concerns the cost-effectiveness of replacing existing paper-based systems with expensive mHealth systems. Earlier research indicates that standard paper-based systems can incur hidden costs in terms of staff time to maintain and correct data entry errors and/or the storage of paper records that are often overlooked when assessing cost-effectiveness (Tomlinson et al., 2009; Holeman \& Nesbit, 2010). Addressing this cost barrier to implementation and scale calls for further evidence-based research into the cost-effectiveness of mHealth interventions compared to traditional paper-based systems and/or hybrid systems which combine electronic and paper-based systems. Establishing the cost-effectiveness of mHealth implementation is indispensable to support the argument for the scale and long-term sustainability of mHealth. 


\subsection{Interoperability: An Open Architecture Framework}

Interoperability is a buzzword that is frequently exchanged at most global forums on mHealth, and much has been documented about the importance of building platforms that can communicate and integrate with existing systems of care as a way to ensure future scale and sustainability (van Heerden, Tomlinson \& Swartz, 2012; PLOS Medicine Editors, 2013). Traditionally health information systems have been built using a silo approach wherein devices and disease-specific applications cannot easily share data with one another. However, there is a growing recognition of the need for improved data integration (De Maeseneer, van Weel, Egilman, Demarzo \& Sewankambo, 2012) alongside interoperable health technology (van Heerden et al., 2012). National-level systems such as the open-source District Health Information Software 2 are currently used in multiple countries for routine health data collection, reporting, and management (DHIS2, n.d.). It is likely that this kind of open source and interoperable mHealth system can have a much greater chance of long-term sustainability rather than parallel siloed solutions. In terms of architecture interoperability, OpenHIE has developed a three-layer health information architecture framework which connects external systems and actors to multiple health datasets via an interoperability services layer (OpenHIE, n.d.). When successfully implemented, these kinds of open architectures can act as an "innovation infrastructure" in the same way as a mobile network or an electricity grid, enhancing the potential power and impact of mHealth systems (Estrin \& Sim, 2010). Government, industry, and donors need to, therefore, cooperate and adopt an open architecture-based approach to developing and implementing mHealth interventions in order to ensure their success, scale, and long-term sustainability.

\subsection{User-Centered Design}

Allotting time and resources to product and project design for mHealth initiatives can yield positive results as the solution scales from a pilot project up to state, district, or national level (Eckman et al., 2016). Technology is the only component of this design challenge; attention must also be given to the end users of the systemusually a health worker - and how a new tool will help them. Those mHealth projects that incorporate user-centered design principles from the outset can fare better than those that did not (Eckman et al., 2016).

Medic Mobile has employed Human-Centered Design (HCD), a specific user-centered design approach that emphasizes a deep understanding of human capabilities, motivations, concerns, and values as they consistently surface in their daily lives; the reliability of a new technology is determined by the routine actions of users within the system (Bannon, 2011). HCD is more a way of thinking than a defined procedure and can take various shapes based on the context and the methods used to put this approach into practice (Kane, 2016). 


\subsection{Impact}

While the global health community —including NGOs, governments, and donorscontinues to display some enthusiasm for mHealth, the lack of rigorous program evaluations presents a barrier to quality mHealth implementations (PLOS Medicine Editors, 2013; Tamrat \& Kachnowski, 2012). The multiyear timeframe required for this kind of program evaluation means that the technology and systems under investigation may have become obsolete by the time that findings are published (Kumar et al., 2013). Therefore, the field of mHealth may benefit from other forms of supporting evidence via continuous monitoring of program activity and outcomes to inform timely dissemination of lessons learned and best practices. The inclusion of robust monitoring and evaluation components within program design is of utmost importance to scaling mHealth systems (ibid.; Agarwal et al., 2016; Whittaker, Merry, Dorey \& Maddison, 2012).

\subsection{Conclusion: The Path to Scale}

mHealth offers an unprecedented opportunity to reach last mile populations around the world and improve health outcomes in challenging settings. However, mHealth interventions are often complex and messy and the narrative around their scale can attract skepticism. Nevertheless, a number of successful mHealth tools and projects have followed a viable pathway from pilot to scale and lessons from these can guide governments, funders, and private enterprise to shape the future mHealth landscape. Albeit challenging, mHealth scale can be achieved with a favorable overarching framework of infrastructure, regulatory and policy environment, stakeholder partnerships, and financial sustainability; as well as a focus on interoperability, context-appropriate technology, robust user-centered design, a skilled health workforce, and an impact-driven approach to mHealth. This checklist offers a potential path to scale that can enable mHealth to fulfill its promise of strengthening systems and improving health outcomes, particularly in low-resource settings.

\section{References}

Adhikari, K. (2015, July 13). Lack of road connection keeps quake-hit Dhading folks in trouble. The Himalayan Times. Retrieved from https://thehimalayantimes.com/nepal/lack-of-roadconnection-keeps-quake-hit-dhading-folks-in-trouble. Accessed 28 Feb 2017.

Agarwal, S., LeFevre, A. E., Lee, J., L’Engle, K., Mehl, G., Sinha, C., et al. (2016). Guidelines for reporting of health interventions using mobile phones: Mobile health (mHealth) evidence reporting and assessment (mERA) checklist. BMJ, 352, i1174. 
Airtel. (2016). Customer Churn. Retrieved from iCreate website. http://www.airtel.in/icreate/ common/files/iCreate_Finance_case_study_2016.pdf. Accessed 5 Jan 2017.

Aranda-Jan, C. B., Mohutsiwa-Dibe, N., \& Loukanova, S. (2014). Systematic review on what works, what does not work and why of implementation of mobile health (mHealth) projects in Africa. BMC Public Health, 14(1), 188.

Bannon, L. (2011). Reimagining HCI: Toward a more human-centered perspective. Interactions, $18(4), 50-57$.

Ben-Zeev, D., Schueller, S. M., Begale, M., Duffecy, J., Kane, J. M., \& Mohr, D. C. (2015). Strategies for mHealth research: Lessons from 3 mobile intervention studies. Administration and Policy in Mental Health and Mental Health Services Research, 42(2), 157-167.

Bernhardsen, T. (1999). Choosing a GIS. Geographical. Information Systems, 2, 589-600.

Bjornland, D., Goh, E., Haanæs, K., Kainu, T., \& Kennedy, S. (2012). The Socio-economic impact of mobile health. The Boston Consulting Group.

Chang, L. W., Kagaayi, J., Arem, H., Nakigozi, G., Ssempijja, V., Serwadda, D., ...Reynolds, S. J. (2011). Impact of a mHealth intervention for peer health workers on AIDS care in rural Uganda: A mixed methods evaluation of a cluster-randomized trial. AIDS and Behavior, 15(8) (1776).

Chetley, A., (Ed.). Davies, J., Trude, B., McConnell, H., Ramirez, R., Shields, T., ... Nyamai-Kisia, C. (2006). Improving health, connecting people: The role of ICTs in the health sector of developing countries-A framework paper. InfoDev Working Paper, no. 7. Health. Washington, DC: World Bank. Retrieved from http://documents.worldbank.org/ curated/en/234041468163474585/Improving-health-connecting-people-the-role-of-ICTs-in-thehealth-sector-of-developing-countries-a-framework-paper.

De Maeseneer, J., van Weel, C., Egilman, D., Demarzo, M., \& Sewankambo, N. (2012). Tackling NCDs: A different approach is needed-authors' reply. The Lancet, 379(9829), 1873-1874.

DHIS2 (n.d.). DHIS2. Retrieved from (DHIS2) District Health Information Software website. http://dhis2.org. Accessed 4 Jan 2017.

Eckman, M., Gorski, I., \& Mehta, K. (2016). Leveraging design thinking to build sustainable mobile health systems. Journal of Medical Engineering \& Technology, 40(7-8), 422-430.

Estrin, D., \& Sim, I. (2010). Open mHealth architecture: An engine for health care innovation. Science, 330(6005), 759-760.

Eysenbach, G. (2009). Infodemiology and infoveillance: Framework for an emerging set of public health informatics methods to analyze search, communication and publication behavior on the internet. Journal of Medical Internet Research, 11(1), e11.

GSMA. (2015). Bridging the gender gap: Mobile access and usage in low and middle-income countries. Retrieved from Group Speciale Mobile Association (GSMA) website. http://www. gsma.com/mobilefordevelopment/wp-content/uploads/2016/02/Connected-Women-Gender-Gap. pdf. Accessed 3 Jan 2017.

Haberer, J. E., Kiwanuka, J., Nansera, D., Wilson, I. B., \& Bangsberg, D. R. (2010). Challenges in using mobile phones for collection of antiretroviral therapy adherence data in a resource-limited setting. AIDS and Behavior, 14(6), 1294-1301.

Hall, C. S., Fottrell, E., Wilkinson, S., \& Byass, P. (2014). Assessing the impact of mHealth interventions in low-and middle-income countries-What has been shown to work? Global Health Action, 7.

Holeman, I., \& Nesbit, J. (2010). mHealth basics and human scalability. Harvard College Global Health Review, 11(1), 40-43.

Iluyemi, A., \& Briggs, J. S. (2008). Technology matters!: Sustaining eHealth in developing countries: Analyses of mHealth innovations. Institution of Engineering and Technology (IET).

International Telecommunications Union (2015, May). ICT facts \& figures: The world in 2015, pp. 1-6. Retrieved from https://www.itu.int/en/ITUD/Statistics/Documents/facts/ICTFacts Figures2015.pdf. Accessed 5 Jan 2017. 
Kane, D. (2016, March 31). Medic mobile's human-centered design toolkit: A spotlight on sketch cards [Blog post]. Medic Mobile Blog. Retrieved from http://medicmobile.org/blog/medicmobiles-human-centered-design-toolkit-a-spotlight-on-sketch-cards. Accessed 8 Jan 2017.

Kay, M., Santos, J., \& Takane, M. (2011). mHealth: New horizons for health through mobile technologies. World Health Organization, 3, 66-71.

Khanal, V., Khanal, P., \& Lee, A. H. (2015). Sustaining progress in maternal and child health in Nepal. The Lancet, 385(9987), 2573.

Kumar, S., Nilsen, W.J., Abernethy, A., Atienza, A., Patrick, K., Pavel, M., ...Hedeker, D. (2013). Mobile health technology evaluation: The mHealth evidence workshop. American Journal of Preventive Medicine, 45(2), 228-236.

Lehmann, U., \& Sanders, D. (2007). Community health workers: What do we know about them. The state of the evidence on programmes, activities, costs and impact on health outcomes of using community health workers (pp. 1-42). Geneva: World Health Organization.

Lemaire, J. (2011). Scaling up mobile health: Elements necessary for the successful scale up of $m$ Health in developing countries. Geneva: Advanced Development for Africa.

Leon, N., Schneider, H., \& Daviaud, E. (2012). Applying a framework for assessing the health system challenges to scaling up mHealth in South Africa. BMC Medical Informatics and Decision Making, 12(1), 123.

Lester, R.T., Ritvo, P., Mills, E.J., Kariri, A., Karanja, S., Chung, M.H., ...Marra, C.A. (2010). Effects of a mobile phone short message service on antiretroviral treatment adherence in Kenya (WelTel Kenya 1): A randomised trial. The Lancet, 376(9755), 1838-1845.

Mechael, P., Batavia, H., Kaonga, N., Searle, S., Kwan, A., Goldberger, A., ...Ossman, J. (2010). Barriers and gaps affecting mHealth in low and middle-income countries: Policy white paper (pp. 1-79). Columbia University. Earth Institute. Center for Global Health and Economic Development (CGHED): With mHealth Alliance.

Mills, A. (2014). Health care systems in low- and middle-income countries. New England Journal of Medicine, 370(6), 552-557.

Modi, D., Gopalan, R., Shah, S., Venkatraman, S., Desai, G., Desai, S., \& Shah, P. (2015). Development and formative evaluation of an innovative mHealth intervention for improving coverage of community-based maternal, newborn and child health services in rural areas of India. Global Health Action, 8.

Nesbit, J. (2015, June 23). Response and rebuilding health systems in Nepal [Blog post]. Medic Mobile Blog. Retrieved from http://medicmobile.org/blog/nepal-earthquake-how-you-can-help. Accessed 28 Feb 2017.

Ngabo, F., Nguimfack, J., Nwaigwe, F., Mugeni, C., Muhoza, D., Wilson, D.R., ...Binagwaho, A. (2012). Designing and implementing an innovative SMS-based alert system (RapidSMS-MCH) to monitor pregnancy and reduce maternal and child deaths in Rwanda. Pan African Medical Journal, 13(31).

OpenHIE (n.d.). Architecture. Retrieved from Open Health Information Exchange (OHIE) website ohie.org/architecture/. Accessed 4 Jan2017.

PLOS Medicine Editors (2013). A reality checkpoint for mobile health: Three challenges to overcome. PLoS Med, 10(2), e1001395.

Qiang, C. Z., Yamamichi, M., Hausman, V., \& Altman, D. (2011). Mobile applications for the health sector. Washington: World Bank.

Rajput, Z. A., Mbugua, S., Amadi, D., Chepnǵeno, V., Saleem, J. J., Anokwa, Y., ...Were, M.C. (2012). Evaluation of an android-based mHealth system for population surveillance in developing countries. Journal of the American Medical Informatics Association, 19(4), $655-659$.

Sanner, T. A., Roland, L. K., \& Braa, K. (2012). From pilot to scale: Towards an mHealth typology for low-resource contexts. Health Policy and Technology, 1(3), 155-164. 
Sharma, R., Harsha, A., Acharya, P., Okada, E., Yangdol, T., Bhatta, S., ...Dahal, S. (2015). Pilot and evaluation of the feasibility SafeSIM: A mobile technology platform for maternal health care coordination in Nepal. Publication Timeline: TBD.

Shaw, V. (2012). Measuring the impact of e-health. Bulletin of the World Health Organization, 90, 326-327.

Strachan, D. L., Källander, K., ten Asbroek, A. H., Kirkwood, B., Meek, S. R., Benton, L., ...Hill, Z. (2012). Interventions to improve motivation and retention of community health workers delivering integrated community case management (iCCM): Stakeholder perceptions and priorities. The American Journal of Tropical Medicine and Hygiene, 87(5), 111-119.

Switchboard. (2017). Work. Retrieved from switchboard.org/work. Accessed 4 Jan 2017.

Tamrat, T., \& Kachnowski, S. (2012). Special delivery: An analysis of mHealth in maternal and newborn health programs and their outcomes around the world. Maternal and Child Health Journal, 16(5), 1092-1101.

The Economist. (2014, April 5). The rise of the cheap smartphone. The Economist. Retrieved from http://www.economist.com/news/business/21600134-smartphones-reach-masses-host-vendorsare-eager-serve-them-rise-cheap. Accessed 5 Jan 2017.

Thondoo, M., Strachan, D. L., Nakirunda, M., Ndima, S., Muiambo, A., Källander, K., ...InSCALE Study Group (2015). Potential roles of Mhealth for community health workers: Formative research with end users in Uganda and Mozambique. JMIR mHealth and uHealth, 3(3).

Timm, T. (2014, May 3). Technology law will soon be reshaped by people who don't use email. The Guardian. Retrieved from theguardian.com/commentisfree/2014/may/03/technology-lawus-supreme-court-internet-nsa. Accessed 9 Jan 2017.

Tomlinson, M., Solomon, W., Singh, Y., Doherty, T., Chopra, M., Ijumba, P., ...Jackson, D. (2009). The use of mobile phones as a data collection tool: A report from a household survey in South Africa. BMC Medical Informatics and Decision Making, 9(1), 51.

Tomlinson, M., Rotheram-Borus, M. J., Swartz, L., \& Tsai, A. C. (2013). Scaling up mHealth: Where is the evidence? PLoS Med, 10(2), e1001382.

Umali, E., McCool, J., \& Whittaker, R. (2016). Possibilities and expectations for mHealth in the Pacific Islands: Insights from key informants. JMIR mHealth and uHealth, 4(1).

van Heerden, A., Tomlinson, M., \& Swartz, L. (2012). Point of care in your pocket: A research agenda for the field of m-health. Bulletin of the World Health Organization, 90(5), 393-394.

Waugaman, A. (2016). From principle to practice: Implementing the principles for digital development. Perspectives and Recommendations from the Practitioner Community. Washington, DC: The Principles for Digital Development Working Group, 1-76. Retrieved from http://www.unicefstories.org/wp-content/uploads/2013/08/From_Principle_to_Practice. pdf.

Whittaker, R., Merry, S., Dorey, E., \& Maddison, R. (2012). A development and evaluation process for mHealth interventions: Examples from New Zealand. Journal of Health Communication, 17(sup1), 11-21.

World Bank (2011, June 21). World Bank supports cross-border energy cooperation between India and Nepal. [Press release]. World Bank. Retrieved from http://www.worldbank.org/en/news/ press-release/2011/06/21/world-bank-supports-cross-border-energy-cooperation-betweenindia-and-nepal. Accessed 4 Jan 2017.

World Bank (2016a). World Bank open data: Mobile cellular subscriptions (per 100 people). Retrieved from http://data.worldbank.org/indicator/IT.CEL.SETS.P2 . Accessed 5 Jan 2017.

World Bank (2016b). World Bank open data: Access to electricity (\% of population). Retrieved from http://data.worldbank.org/indicator/EG.ELC.ACCS.ZS. Accessed 5 Jan 2017.

World Health Organization (2016). Global diffusion of eHealth: Making universal health coverage achievable. Report of the third global survey on eHealth. Geneva: World Health Organization. Retrieved from http://who.int/goe/publications/global_diffusion/en/. Accessed 7 Jan 2017.

Zurovac, D., Sudoi, R. K., Akhwale, W. S., Ndiritu, M., Hamer, D. H., Rowe, A. K., et al. (2011). The effect of mobile phone text-message reminders on Kenyan health workers' adherence to malaria treatment guidelines: A cluster randomised trial. The Lancet, 378(9793), 795-803. 
Open Access This chapter is licensed under the terms of the Creative Commons Attribution 4.0 International License (http://creativecommons.org/licenses/by/4.0/), which permits use, sharing, adaptation, distribution and reproduction in any medium or format, as long as you give appropriate credit to the original author(s) and the source, provide a link to the Creative Commons license and indicate if changes were made.

The images or other third party material in this chapter are included in the chapter's Creative Commons license, unless indicated otherwise in a credit line to the material. If material is not included in the chapter's Creative Commons license and your intended use is not permitted by statutory regulation or exceeds the permitted use, you will need to obtain permission directly from the copyright holder.

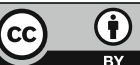

